\title{
BMJ Open Acupuncture for cancer pain: protocol for a pilot pragmatic randomised controlled trial
}

\author{
Yihan $\mathrm{He},{ }^{1,2,3}$ Brian $\mathrm{H}$ May, ${ }^{2}$ Anthony Lin Zhang, ${ }^{2}$ Xinfeng Guo, ${ }^{1,2,3}$ Yihong Liu, ${ }^{1,2,3}$ \\ Yanchun Qu, ${ }^{1,2,3}$ Xuesong Chang ${ }^{3}$ Chuan-jian Lu, ${ }^{1,2,3}$ Charlie Changli Xue, ${ }^{1,2,3}$ \\ Haibo Zhang ${ }^{1,2,3}$
}

To cite: He Y, May BH, Zhang AL, et al. Acupuncture for cancer pain: protocol for a pilot pragmatic randomised controlled trial. BMJ Open 2019;9:e025564. doi:10.1136/ bmjopen-2018-025564

- Prepublication history and additional material for this paper are available online. To view please visit the journal (http:// dx.doi.org/10.1136/bmjopen2018-025564).

Received 24 July 2018 Revised 27 February 2019 Accepted 13 June 2019

Check for updates

(C) Author(s) (or their employer(s)) 2019. Re-use permitted under CC BY-NC. No commercial re-use. See rights and permissions. Published by BMJ.

${ }^{1}$ Guangdong Provincial Hospital of Chinese Medicine (The Second Affiliated Hospital of Guangzhou University of Chinese Medicine), Guangdong Provincial Academy of Chinese Medical Science, Guangzhou,

Guangdong, China

${ }^{2}$ China-Australia International Research Centre for Chinese Medicine, School of Health and Biomedical Sciences, RMIT University, Melbourne, Victoria, Australia

${ }^{3}$ The Second Clinical College, Guangzhou University of Chinese Medicine, Guangzhou, Guangdong, China

Correspondence to

Dr Charlie Changli Xue; charlie.xue@rmit.edu.au and Professor Haibo Zhang;

haibozh@aliyun.com

\section{ABSTRACT}

Introduction Although acupuncture has been recommended for alleviating cancer pain by clinical guidelines, the level of the supporting evidence needs to be improved. A pragmatic randomised controlled trial (pRCT) in a hospital setting would provide real-world assessments of the overall clinical effects of acupuncture. This pilot trial aims to explore the feasibility and provide data for sample size calculations for a pRCT evaluating the effectiveness of acupuncture as an adjunctive therapy to routine medical care for cancer pain.

Methods and analysis Thirty patients with cancer admitted to the oncology department with moderate or severe pain will be recruited. Participants will be randomised at a ratio of 1:1 to the adjunctive acupuncture group or a control group which receives routine pain management without acupuncture. The standardised section of the acupuncture protocol will be developed based on the results of reviews of the literature, recommendations in clinical guidelines and interviews with clinical experts. The acupuncturist will be allowed to tailor the protocol according to the individual situation of each participant. Primary outcomes relevant to the feasibility of conducting a fully powered trial include: numbers and proportions of participants recruited, screened, consented and randomised; numbers and reasons for withdrawals and dropouts; numbers and types of adverse events; feasibility of implementing the trial procedures; evaluation of the comprehensiveness and ease-of-use of the case report form. Secondary outcomes are clinical measurements of the effectiveness of the treatment that are intended for use in the full-scale trial. Analysis of feasibility will be descriptive and pain intensity measures will be analysed using mixed-effects regression.

Ethics and dissemination Ethics approval was obtained from the Institutional Ethics Committee of Guangdong Provincial Hospital of Chinese Medicine (approval no: Z2017-184-01) and RMIT University Human Research Ethics Committee (reference no: 21361). Results will be disseminated in a peer-reviewed journal, and trial participants will be informed via email and/or phone calls. Trial registration number ChiCTR1800017023

\section{BACKGROUND}

Pain is a common clinical symptom in patients with cancer with a prevalence rate

\section{Strengths and limitations of this study}

- The comprehensive literature research and consultation with clinicians will contribute to the rationality and practicability of the standardised section of the acupuncture protocol.

- The permission to partially tailor acupuncture points to the needs of each participant will increase the external validity of the acupuncture protocol.

- The results of this feasibility study will provide data for an adequately powered pragmatic trial.

- Mixed-effects regression analyses will discriminate possible differences due to baseline characteristics and intervention variations.

- The results of this feasibility study will not be able to determine treatment effectiveness.

of over $70 \%$ for malignancies. ${ }^{1}$ Nearly half of patients with cancer rated their pain severity as moderate to severe (Numerical Rating Scale, NRS $\geq 5) .{ }^{23}$ Pain has a serious impact on activities of daily living and quality of life for patients with cancer, ${ }^{4}$ and pain relief has been highly advocated as an imperative of universal health coverage. ${ }^{5}$

The widely adopted WHO three-step analgesic ladder ${ }^{6}$ is effective in controlling pain for patients with cancer; however, unavailability of opioids, ${ }^{7}$ non-adherence to prescriptions ${ }^{8}$ and the risks of long-term adverse effects $^{9}$ are still obstacles to the application of these methods. The National Comprehensive Cancer Network (NCCN) guideline for adult cancer pain recommends comprehensive and individualised strategies in pain management including pharmacological and non-pharmacological interventions. ${ }^{10}$ Acupuncture has been considered as one of the physical modalities to relieve pain and improve function for patients with cancer since the early version of the NCCN cancer pain guideline. ${ }^{11}$

The role of acupuncture for cancer pain has been supported by evidence from 
experimental studies, clinical trials and clinical experience, and acupuncture has been suggested as an auxiliary to standard treatments. ${ }^{12} \mathrm{~A}$ rigorous large-scale randomised controlled trial published recently reported that true acupuncture, compared with sham acupuncture or waitlist control, resulted in a significant reduction in aromatase inhibitor-related joint pain among women with breast cancer. ${ }^{13}$

However, the use of sham control methods for acupuncture remains a field filled with disputation. Sham acupuncture is argued to be an inappropriate control because of its potential physiological effects ${ }^{14-17}$ and the difficulty in masking ${ }^{18} 19$ due to the nature of sham acupuncture. ${ }^{20} 21$ In real-world clinical settings, acupuncture is usually used as an adjuvant therapy to conventional care. Hence, comparing acupuncture alone with standard care may have limited external validity. ${ }^{22}$ Pragmatic randomised controlled trials (pRCTs) that compare acupuncture plus standard care versus standard care alone may provide results that can directly inform clinical practice. ${ }^{17}$

Pragmatic trials emphasise practical applicability and extrapolation to real-world situations (external validity) and usually focus on the overall effects rather than on the efficacy of the treatment. ${ }^{23}$ They are particularly appropriate for research on complex and flexible interventions with subjective outcomes. ${ }^{23-25}$ It has been suggested that pragmatic trials in real-world settings could be used to investigate the effectiveness of acupuncture. ${ }^{26} 27$

\section{Objectives}

A pilot trial is highly recommended as component of protocol development to assess the feasibility and capacity to conduct a fully powered study. ${ }^{28} 29$

The primary aim of this pilot trial is to evaluate the feasibility of a prospective pRCT for the effectiveness of acupuncture as an adjunctive therapy to routine medical care for cancer pain. Results from this pilot trial will optimise study design and provide data for sample size calculation of the definitive trial.

The specific objectives are to:

- Investigate the proportions of participants confirmed as screened, eligible and consenting (including reasons for exclusion), and the time required to recruit the target number of participants.

- Explore the effectiveness of the methods and procedures for random allocation and methods for blinding outcome assessment.

- Determine the numbers and reasons for withdrawals and dropouts, and numbers and types of adverse events.

- Assess the feasibility of the instruments and procedures for outcome measurement and data collection.

- Explore factors that potentially impact outcome measures, such as type and stage of cancer, category and degree of pain at baseline, variation in interventions and response expectancy of participants for acupuncture.

\section{METHODS}

\section{Trial design}

Unlike most traditional randomised controlled trials which test a hypothesis under ideal conditions or determine the effects of a specific treatment, pRCTs compare treatments under everyday clinical conditions, with the aim of improving practice by informing clinical and policy decisions directly. They are characterised by more representative samples because there is less restriction on eligibility criteria, real-world treatments using flexible protocols and local customisation, and brief outcome assessments so data can be easily collected in clinical settings. ${ }^{24}$

A pilot pRCT will be conducted to evaluate the feasibility of conducting a fully powered pRCT of acupuncture as an adjunctive therapy to routine medication and usual care for cancer pain. The study design will incorporate individualised treatment flexibility, as in a real-world setting, which is expected to provide realistic estimates of acupuncture effects ${ }^{28}$ when implemented in a fully powered pRCT. The study procedures is summarised in the CONSORT (Consolidated Standards of Reporting Trials) diagram (figure 1).

\section{Participants}

\section{Eligibility criteria}

Consistent with the design of a pragmatic trial, the inclusion criteria are intentionally broad to reflect the heterogeneity of clinical practice. ${ }^{31}$

Eligible participants will be:

- Patients admitted to the oncology inpatient department for an anticipated length of stay of at least 1 week.

- 18 years of age or older.

- With confirmed pathological diagnosis of cancer.

- Suffering from moderate or severe cancer pain $(\mathrm{NRS} \geq 4)^{10}$ at admission or during the stay.

- Current use of analgesic medication is allowed.

Exclusion criteria will include:

- Contraindication to acupuncture (eg, extreme needle phobia or severe clotting dysfunction).

- Incapacity to consent (eg, cognitive or communication impairment).

- Severe and unstable clinical disorders (eg, myocardial infarction, neutropenia). ${ }^{32}$

\section{Recruitment and withdrawal}

The study will be conducted at the Oncology Department of Guangdong Provincial Hospital of Chinese Medicine (GPHCM), which is a 45-bed inpatient unit that provides specialist care for patients with cancer and has well-established protocols for pain management. All admitted patients who meet initial eligibility criteria will be asked if they are willing to participate in this study. All participants who consent to the study will be requested to sign the informed consent. 


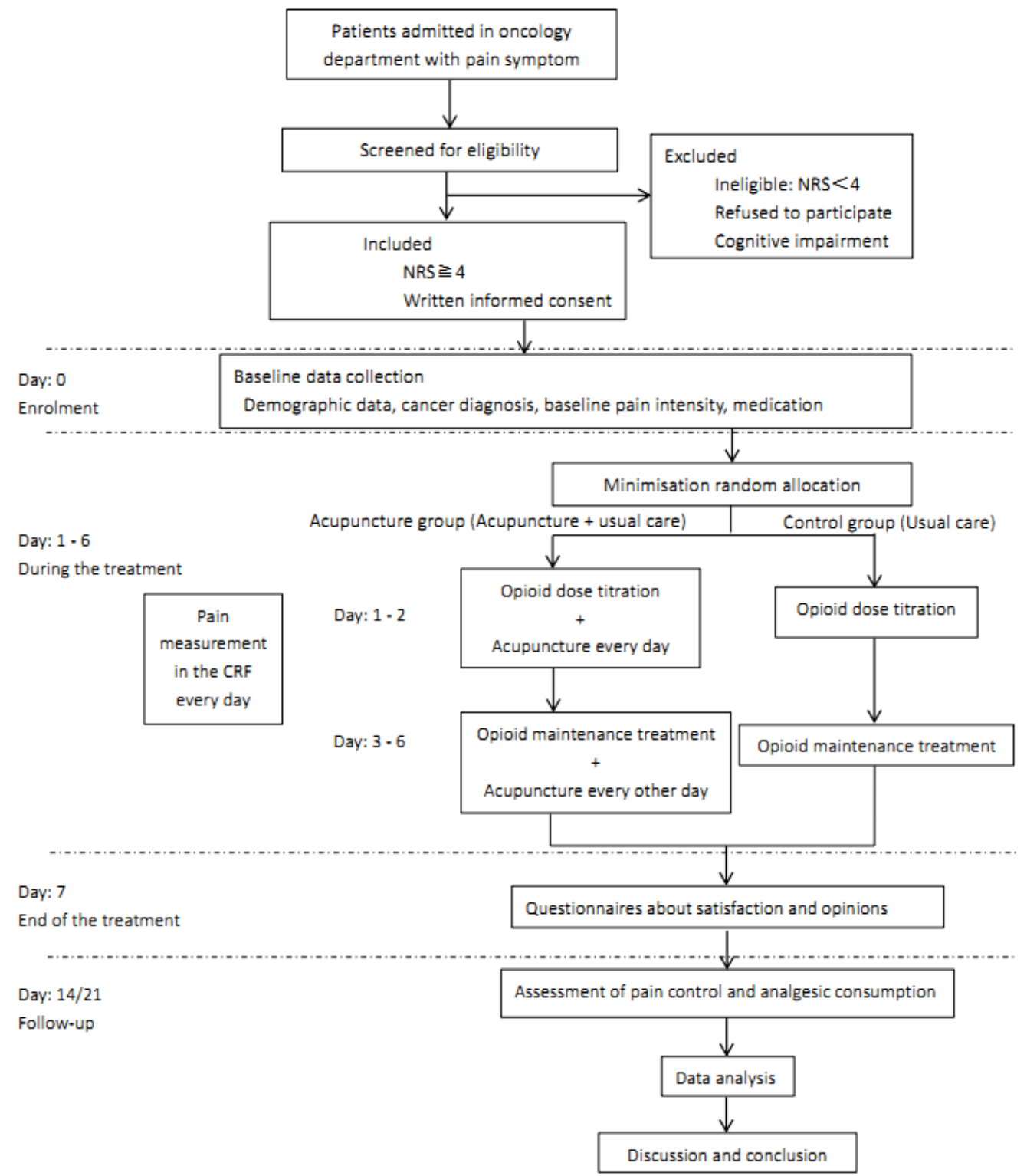

Figure 1 Trial procedure flow chart. CRF, case report form; NRS, Numerical Rating Scale.

Participants are permitted to withdraw at any time during the trial, with or without reasons being provided. All withdrawn cases who have received the intervention will be contacted for a 2-week follow-up period to obtain information in regard to their condition.

\section{Sample size}

Since the study is a pilot study, a formal sample size calculation is not required. ${ }^{33}$ One analysis identified 12 participants per group as an appropriate sample in a feasibility study. ${ }^{34}$ Another study calculated that power could be adequate for moderate effect sizes at 15 per group. ${ }^{35}$ Considering a $25 \%$ dropout rate, ${ }^{36}$ we selected 15 participants per group for this pilot trial. Considering the short duration (3 weeks) of the proposed study and the high degree of cultural acceptability of acupuncture in the proposed participant population, it appears likely that the dropout rate could be lower than $25 \% .{ }^{37}$

\section{Randomisation and blinding}

Minimisation random allocation will be applied to ensure balance between the intervention group and the control group for several prognostic factors, ${ }^{38}$ such as gender, age, type and stage of cancer. An independent staff member from the Key Unit of Methodology in Clinical Research of GPHCM will carry out the randomisation by the web-based minimization random allocation system, MinimRan. ${ }^{39}{ }^{40}$ Although there is dispute on whether minimisation precludes allocation concealment, ${ }^{41}{ }^{42}$ both the statistical and clinical validity of minimisation could be achieved by proper approaches to statistical analysis. $^{434}$

Participants and practitioners involved in the study will not be blinded. However, the researchers collecting primary data and performing statistical analysis will be blinded to the participant's allocation. The success of 
blinding for assessors will be assessed by the answers to the question: "which group do you believe this participant belongs to?'

\section{Intervention}

In addition to routine pain management and usual care, participants will receive acupuncture treatment.

\section{Acupuncture treatment}

One licensed acupuncturist with more than 3years' acupuncture experience will provide acupuncture treatment to all participants. The acupuncturist will not be, or have been, involved in any other aspect of the treatment of the participants. Apart from acupuncture, other therapies related to stimulating acupuncture points such as acupressure, moxibustion and transcutaneous electrical nerve stimulation are not allowed during this trial.

Disposable pads with $70 \%$ isopropyl alcohol will be used to sterilise selected acupuncture points. Single-use, sterilised needles made of stainless steel (Suzhou Huanqiu Acupuncture Medical Instrument, Suzhou, China) will be inserted to a depth of $15-35 \mathrm{~mm}$ in the sterilised acupuncture points. Needle insertion should be gentle and the depth of insertion may vary according to individual condition and point position. The acupuncturist will manipulate the inserted needles until the participant feels acceptable and comfortable but intense sensations. Needles will be left in place for $30 \mathrm{~min}$.

The standardised section of the acupuncture protocol has been developed by expert consensus based on the most frequently used and effective acupuncture points in premodern literature and clinical trials of acupuncture for cancer pain. Twenty-two acupuncturists from the China Association of Acupuncture and Moxibustion and three international acupuncturists with extensive acupuncture experience participated in the survey. Significant agreement was achieved on the necessity of individualised acupuncture protocols and the effectiveness of Hegu (LI4), Taichong (LR3), Zusanli (ST36), Sanyinjiao (SP6) and Yanglingquan (GB34) in pain alleviation (online supplementary 1 ). Therefore, all participants will be treated on the five acupuncture points above. Additionally, the acupuncturist will be allowed to add up to five more acupuncture points according to the individual conditions and symptoms of each participant. Individualised acupuncture points will be chosen based on the principles of Traditional Chinese Medicine, including local points around the location(s) with the most pain and distant points addressing constitutional symptoms. The acupuncturist will be trained in the use of the semistandardised protocol, reporting adverse events and other trial procedures. ${ }^{45}$

Participants will receive four sessions of acupuncture treatment in total, namely every day in the first 2 days of the trial (on day $1 / 2$ ) and once every 2 days during the remainder of the trial (on day 4/6). Acupuncture will be delivered during 14:00-15:00 without administration of acesodyne. The duration of the treatment course will be 1 week from the day after they are enrolled into the study. The whole process of acupuncture treatment will be supervised by another researcher for double-checking the acupuncture point selection and standard operating procedure. Details of acupuncture therapies (eg, number and specification of needles used, specific acupuncture points applied and duration of the therapies) will be recorded in the acupuncture treatment record section of the case report form (CRF, online supplementary 2) by the acupuncture supervisor and signed off by the acupuncturist.

Although acupuncture is a relatively safe treatment with a low risk of adverse events ${ }^{46}$ any event arising from the acupuncture intervention will be treated immediately and documented in detail. In the event of mild and temporary adverse events, such as bleeding or haematoma, participants will not be withdrawn but will be able to drop out if they wish. In the event of serious adverse events, such as vertigo, palpitations, local infection, a formal report will be submitted to the ethics committees and participants will be withdrawn.

\section{Routine pain management and usual care}

All study participants in both groups will receive usual care, as is the regular practice of the oncology department. They will receive education on palliative care, the ongoing process of pain assessment and the customised strategies for pain control. Routine pain management will be based on the NCCN guidelines. ${ }^{10}$ If pain cannot be controlled within 48 hours after pain management, specialty consultation will be considered and the participant will be withdrawn. Health professionals have adequate knowledge and skills to provide palliative care practices. National and hospital policies ensure the availability of drugs required for pain management. Adverse events due to opioids will be managed in accordance with the NCCN guideline. Detailed information on the procedures for controlling pain will be collected retrospectively from patients' electronic medical record and transferred to the Analgesic treatment records section in the CRF by the data administrator.

\section{Comparison}

Treatment of control group

Participants in the control group will receive routine pain management and usual care, as outlined above, without acupuncture. They will be allowed to receive acupuncture treatment if required, but these cases will be regarded as withdrawal.

\section{Outcomes}

Primary outcomes include measurements relevant to the feasibility of conducting a large-scale pRCT. Secondary outcomes involve measurements of clinical effectiveness intended for use in the full-scale trial.

\section{Trial feasibility}

Feasibility will be assessed by analysing data collected during the whole process of the trial. The number of patients 
recruited and screened, the number meeting the inclusion criteria and the number agreeing to participate in the trial will be used to calculate the proportion of involvement to assess the feasibility of the recruitment process. Participants who withdraw and decline further contact, including participants who have withdrawn after randomisation but prior to receiving the intervention are defined as dropouts. Data from dropout cases will be managed by both per-protocol (PP) analysis and intention-to-treat (ITT) analysis to evaluate the influence of missing data.

The feasibility of the methods used for randomisation and allocation concealment and any challenges encountered will be assessed by interviewing the staff member from the Key Unit of Methodology in Clinical Research of GPHCM who conducted these procedures.

The effectiveness of the blinding of outcome assessors will be assessed using a questionnaire.

\section{Clinical outcomes}

Participant-reported pain intensity will be measured by the NRS, ${ }^{47}$ an instrument recommended by the European Society for Medical Oncology guideline ${ }^{48}$ that shows reliable sensitivity and validity to assess pain. ${ }^{49}{ }^{50}$ Analgesic consumption, frequency of breakthrough pain and rescue medication used, and adverse events will also be clinical outcome measurements. These are recorded in the CRF. In addition, response expectancy of participants for acupuncture will be measured by the Acupuncture Expectancy Scale. ${ }^{51}$

\section{Data collection}

Participant's demographic data (age, sex, nationality, height, weight), cancer diagnosis (site, stage, pathology), baseline pain intensity, pain management situation if any (current regular pain medication dosage, rescue medication dosage in the 1 week previous to being enrolled in the study, and adverse events) will be collected when they are enrolled. An independent data administrator blinded to the group allocation and not involved in the treatment will collect individual data accurately into the Pain assessment records in the CRF. Pain intensity ratings will be obtained every $60 \mathrm{~min}$ for participants taking oral opioids or every $15 \mathrm{~min}$ for those receiving intravenous bolus during opioid titration. When pain is stable over time, participants will be asked to assess the pain intensity twice a day, namely early in the morning at 08:00 and late in the afternoon at 18:00. ${ }^{52}$ The time of acupuncture treatment and pain assessment will be staggered to ensure the blindness of the data administrator. Analgesic consumption, frequency of breakthrough pain and rescue medication used, adverse events and withdrawals or dropouts for any reason will be recorded in the Analgesic treatment records, Records of Medications and Other Therapies and Reports of Adverse Events in the CRF by the data administrator. It is only information about adjunctive pain management/usual care that will be collected retrospectively and not pain intensity ratings used as an outcome measure over the trial period.
The follow-up of this trial will be 2 weeks. A short follow-up period was chosen because short-term acupuncture treatment is believed to have a relatively short-lived effect and confounding factors are considerable and uncontrollable after discharge. During this period, participants are required to record their pain intensity, analgesic consumption, frequency of breakthrough pain and rescue medication used, as well as adverse events in the Follow-up records in the CRF every day. Participants will be contacted by telephone every day as a reminder. Their follow-up CRFs will be collected at the end of the follow-up.

\section{Statistical analysis}

The primary analysis of this pilot trial will focus on the outcomes assessing feasibility by descriptive analysis. The number and the proportion of participants recruited, screened, consented and randomised will be presented. The characteristics of participants, number and reasons for withdrawals from the trial, and adverse events will be summarised for each group.

Exploratory analysis of the effect sizes for pain intensity will be conducted by mixed-effects regression analyses, controlling for significant baseline characteristics, such as gender, age, type and stage of cancer, category and degree of inceptive pain, and major distinctions with regard to treatments of acupuncture or analgesics. Since some participants who agreed to accept acupuncture treatment and complete treatment may not receive any acupuncture treatment or withdraw for various reasons, both PP analysis and ITT analysis will be undertaken to detect any discrepancy and determine the robustness of the result.

All data entry will use anonymous sheets and statistical analysis will be undertaken by an independent statistician blinded to treatment allocation using the Statistical Package for the Social Sciences.

\section{Patient and public involvement}

The research question on the effect of acupuncture for cancer pain was first proposed by a patient in the oncology department who could not receive routine pain management due to insufficient pain control and unbearable side effects. Patients were not involved in developing or implementing the study.

\section{Ethics and dissemination}

This study has been approved by the Institutional Ethics Committee of Guangdong Provincial Hospital of Traditional Chinese Medicine (approval No: Z2017-184-01) and the RMIT Human Research Ethics Committee (reference No: 21361). All participants will be provided and asked to sign the informed consent (online supplementary 3$)$.

The results of this study will be submitted for publication in peer-reviewed publications. The results will also be disseminated to study participants via email and/or phone calls. 


\section{DISCUSSION}

The effectiveness, safety and acceptance by the patients of acupuncture in previous studies suggested the feasibility and necessity of conducting a pRCT of acupuncture for cancer pain. A single-arm pragmatic pilot study at the University of Texas MD Anderson Cancer Center showed that 41 patients with cancer with moderate to severe pain had significant improvement in pain and took less prescription pain medication after acupuncture therapies. ${ }^{53}$ The study adopted a combination acupuncture protocol of auricular points (unified selected points) and body points (individualised selected points), and the selection of auricular points was based on a previous study using ear and scalp acupuncture for blast-injured US military service members with limb loss ${ }^{54}$ while the body acupuncture points were determined by the acupuncturist according to the theory of traditional Chinese medicine. Another single-arm pragmatic feasibility trial was conducted in the Abramson Cancer Center of the University of Pennsylvania and the results indicated that individualised electroacupuncture could improve symptoms related to aromatase inhibitor-induced arthralgia for breast cancer survivors. ${ }^{55}$ The semistandardised acupuncture protocol was developed by referring to previous clinical trials on acupuncture for related diseases such as osteoarthritis, ${ }^{56}$ postmenopausal hot flashes ${ }^{5758}$ and endometriosis-related pelvic pain, ${ }^{59}$ and additionally by consulting the faculty of an institute of acupuncture and moxibustion. ${ }^{55}$ In light of the above trials, the process of selecting acupuncture points for the proposed study could benefit from the integration of experience from previous studies and consensus from clinical experts.

In this proposed study, the selection of acupuncture points will be on the basis of comprehensive research including: text mining of premodern Chinese medical literature, ${ }^{60}$ a systematic review of contemporary clinical trials ${ }^{61}$ and consultation with clinicians actively involved in providing acupuncture for cancer pain via questionnaires. This protocol will allow the acupuncture procedure to be as consistent with the way acupuncture is typically performed in clinical practice, and aims to provide instruction for future research and clinical application. Another merit of this study is the broad inclusion criteria. Except for restricting pain severity to moderate to severe, there are no other limitations on eligibility. Therefore, this trial could be as close to the actual clinical situation as possible so as to meet the strong extrapolation requirements of pRCTs. ${ }^{62}$

However, there are some limitations in this study. The minimisation of inclusion and exclusion criteria, the potential diversity of individualised treatments, and participants' experience and expectancy of acupuncture may introduce additional variables which may affect the outcomes. ${ }^{63}$ A mixed-effects regression will be employed in analysing the primary outcome, but considering the relatively small sample size of this pilot trial there may be insufficient statistical power to determine the effects of all variables. In addition, since there will be only four acupuncture sessions in the 1-week treatment course, participants will enter their own data in the CRF during the 2-week follow-up. This may not be an adequate time period to evaluate the effectiveness of acupuncture.

In conclusion, this article presents a rigorous design for a pilot pRCT on acupuncture for cancer pain. The results of this trial will provide an assessment of the feasibility of the protocol and data for power calculations to inform the development of a larger scale trial.

Acknowledgements The authors thank all involved in the study, including practitioners, assessors and participants.

Contributors This protocol was first conceived by $\mathrm{YH}$, with critical contributions from the other authors. YH and XC wrote the first draft of the protocol and submitted the registration on ChiCTR. XG, YL and $Y Q$ provided help to design and edited the manuscript. ALZ, BHM and C-jL revised the manuscript. All authors contributed constructive comments on the manuscript and approved the final paper. $\mathrm{YH}, \mathrm{HZ}$ and CCX are the guarantors for the publication and are fully responsible for the paper.

Funding This trial is supported by the Guangdong Provincial Academy of Chinese Medical Sciences, China, and RMIT University through the China-Australia International Research Centre for Chinese Medicine as well as PhD scholarship support to the first author provided by the School of Health and Biomedical Sciences, RMIT University, the 'Specific Research Fund for TCM Science and Technology of Guangdong Provincial Hospital of Chinese Medicine (2018)' (No: YN2018QL04), and the 'Research Project of Traditional Chinese Medicine Bureau of Guangdong Province (2019)' (No: 20191126). HZ and CCX obtained funding for this study.

Competing interests None declared.

Patient consent for publication Obtained.

Provenance and peer review Not commissioned; externally peer reviewed.

Open access This is an open access article distributed in accordance with the Creative Commons Attribution Non Commercial (CC BY-NC 4.0) license, which permits others to distribute, remix, adapt, build upon this work non-commercially, and license their derivative works on different terms, provided the original work is properly cited, appropriate credit is given, any changes made indicated, and the use is non-commercial. See: http://creativecommons.org/licenses/by-nc/4.0/.

\section{REFERENCES}

1. Neufeld NJ, Elnahal SM, Alvarez RH. Cancer pain: a review of epidemiology, clinical quality and value impact. Future Oncol 2017;13:833-41.

2. van den Beuken-van Everdingen $M H$, Hochstenbach LM, Joosten $\mathrm{EA}$, et al. Update on prevalence of pain in patients with cancer: systematic review and meta-analysis. J Pain Symptom Manage 2016;51:1070-90.

3. Marcus DA. Epidemiology of cancer pain. Curr Pain Headache Rep 2011;15:231-4.

4. Kim YC, Ahn JS, Calimag MM, et al. Current practices in cancer pain management in Asia: a survey of patients and physicians across 10 countries. Cancer Med 2015;4:1196-204.

5. Knaul FM, Farmer PE, Krakauer EL, et al. Alleviating the access abyss in palliative care and pain relief-an imperative of universal health coverage: the Lancet Commission report. Lancet 2018;391.

6. Ventafridda V, Saita L, Ripamonti C, et al. WHO guidelines for the use of analgesics in cancer pain. Int J Tissue React 1985;7:93-6.

7. Ventafridda V, Tamburini M, Caraceni A, et al. A validation study of the WHO method for cancer pain relief. Cancer 1987;59:850-6.

8. Moskovitz BL, Benson CJ, Patel AA, et al. Analgesic treatment for moderate-to-severe acute pain in the United States: patients' perspectives in the Physicians Partnering Against Pain (P3) survey. $J$ Opioid Manag 2011;7:277-86.

9. Bennett M, Paice JA, Wallace M. Pain and opioids in cancer care: benefits, risks, and alternatives. Am Soc Clin Oncol Educ Book 2017;37:705-13.

10. NCCN Clinical Practice Guidelines in Oncology (NCCN Guidelines $®$ ) Adult cancer pain Version 2.2017 - May 10. 2017. https://www.nccn. org/professionals/physician_gls/pdf/pain.pdf

11. Swarm R, Abernethy AP, Anghelescu DL, et al. Adult cancer pain. $J$ Natl Compr Canc Netw 2010;8:1046-86. 
12. Paley CA, Johnson MI. Acupuncture for the management of cancerrelated pain? Pain Manag 2017;7:345-9.

13. Hershman DL, Unger JM, Crew K. Acupuncture for aromatase inhibitor-related joint pain among breast cancer patients. JAMA 2018;320:2270-1.

14. Chien TJ, Liu CY, Chang YF, et al. Acupuncture for treating aromatase inhibitor-related arthralgia in breast cancer: a systematic review and meta-analysis. J Altern Complement Med 2015;21:251-60.

15. Yang GS, Kim HJ, Griffith KA, et al. Interventions for the treatment of aromatase inhibitor-associated arthralgia in breast cancer survivors: a systematic review and meta-analysis. Cancer Nurs 2017;40:E26-E41.

16. Franconi G, Manni L, Schröder S, et al. A systematic review of experimental and clinical acupuncture in chemotherapy-induced peripheral neuropathy. Evid Based Complement Alternat Med 2013;2013:1-7.

17. Paley CA, Johnson MI, Tashani OA, et al. Acupuncture for cancer pain in adults. Cochrane Database Syst Rev 2015;10:CD007753.

18. Chen L, Lin CC, Huang TW, et al. Effect of acupuncture on aromatase inhibitor-induced arthralgia in patients with breast cancer: A meta-analysis of randomized controlled trials. Breast 2017;33:132-8.

19. Pan CX, Morrison RS, Ness J, et al. Complementary and alternative medicine in the management of pain, dyspnea, and nausea and vomiting near the end of life. A systematic review. J Pain Symptom Manage 2000;20:374-87.

20. Lund I, Näslund J, Lundeberg T. Minimal acupuncture is not a valid placebo control in randomised controlled trials of acupuncture: a physiologist's perspective. Chin Med 2009;4:1.

21. Lundeberg T, Lund I, Näslund J, et al. The Emperors sham wrong assumption that sham needling is sham. Acupunct Med 2008;26:239-42.

22. Lau $\mathrm{CH}$, Wu X, Chung VC, et al. Acupuncture and related therapies for symptom management in palliative cancer care: systematic review and meta-analysis. Medicine 2016;95:e2901.

23. Sox HC, Lewis RJ. Pragmatic trials: practical answers to "real world" questions. JAMA 2016;316:1205-6.

24. Ford I, Norrie J. Pragmatic trials. N Engl J Med 2016;375:454-63.

25. Chalkidou K, Tunis S, Whicher D, et al. The role for pragmatic randomized controlled trials ( $\mathrm{pRCTs}$ ) in comparative effectiveness research. Clin Trials 2012;9:436-46.

26. Kaptchuk TJ, Chen KJ, Song J. Recent clinical trials of acupuncture in the West: responses from the practitioners. Chin J Integr Med 2010;16:197-203.

27. Luo J, Xu H, Liu B. Real world research: a complementary method to establish the effectiveness of acupuncture. BMC Complement Altern Med 2015;15:153.

28. Craig P, Dieppe P, Macintyre S, et al. Developing and evaluating complex interventions: the new Medical Research Council guidance. BMJ 2008;337:a1655

29. Worsley SD, Oude Rengerink K, Irving E, et al. Series: pragmatic trials and real world evidence: paper 2. Setting, sites, and investigator selection. J Clin Epidemiol 2017;88:14-20.

30. Witt CM, Aickin M, Baca T, et al. Effectiveness Guidance Document (EGD) for acupuncture research - a consensus document for conducting trials. BMC Complement Altern Med 2012;12:148

31. Patsopoulos NA. A pragmatic view on pragmatic trials. Dialogues Clin Neurosci 2011;13:217-24.

32. Filshie J, Hester J. Guidelines for providing acupuncture treatment for cancer patients--a peer-reviewed sample policy document. Acupunct Med 2006;24:172-82.

33. Thabane L, Ma J, Chu R, et al. A tutorial on pilot studies: the what, why and how. BMC Med Res Methodol 2010;10:11.

34. Julious SA. Sample size of 12 per group rule of thumb for a pilot study. Pharm Stat 2005;4:287-91.

35. Hertzog MA. Considerations in determining sample size for pilot studies. Res Nurs Health 2008:31:180-91.

36. Brady B, Veljanova I, Schabrun S, et al. Integrating culturally informed approaches into the physiotherapy assessment and treatment of chronic pain: protocol for a pilot randomised controlled trial. BMJ Open 2017;7:e014449.

37. Brady B, Veljanova I, Schabrun S, et al. Integrating culturally informed approaches into physiotherapy assessment and treatment of chronic pain: a pilot randomised controlled trial. BMJ Open 2018;8:e021999.

38. Altman DG, Bland JM. Treatment allocation by minimisation. BMJ 2005;330:843.
39. Xiao L, Huang Q, Yank V, et al. An easily accessible Web-based minimization random allocation system for clinical trials. J Med Internet Res 2013;15:e139.

40. DHSR/PAMFRI MinimRan (Beta). http://studies.pamfri.org:8080/ minimRan/index.jsp

41. Berger VW. Minimization, by its nature, precludes allocation concealment, and invites selection bias. Contemp Clin Trials 2010;31:406.

42. Taves DR. Minimization does not by its nature preclude allocation concealment and invite selection bias, as Berger claims. Contemp Clin Trials 2011;32:323.

43. Hagino A, Hamada C, Yoshimura I, et al. Statistical comparison of random allocation methods in cancer clinical trials. Control Clin Trials 2004;25:572-84.

44. Xu Z, Proschan M, Lee S. Validity and power considerations on hypothesis testing under minimization. Stat Med 2016;35:2315-27.

45. Greenlee H, Crew KD, Capodice J, et al. Methods to Standardize a Multicenter Acupuncture Trial Protocol to Reduce Aromatase Inhibitor-related Joint Symptoms in Breast Cancer Patients. $J$ Acupunct Meridian Stud 2015;8:152-8.

46. Witt CM, Pach D, Brinkhaus B, et al. Safety of acupuncture: results of a prospective observational study with 229,230 patients and introduction of a medical information and consent form. Forsch Komplementmed 2009;16:91-7.

47. Mosby M. Pain: clinical manual for nursing practice. Nursing standard 1994:9:55.

48. Ripamonti Cl, Santini D, Maranzano E, et al. Management of cancer pain: ESMO Clinical Practice Guidelines. Ann Oncol 2012;23:vii139-54.

49. Hjermstad MJ, Fayers PM, Haugen DF, et al. Studies comparing Numerical Rating Scales, Verbal Rating Scales, and Visual Analogue Scales for assessment of pain intensity in adults: a systematic literature review. J Pain Symptom Manage 2011;41:1073-93.

50. Ferreira-Valente MA, Pais-Ribeiro JL, Jensen MP. Validity of four pain intensity rating scales. Pain 2011;152:2399-404.

51. Mao JJ, Armstrong K, Farrar JT, et al. Acupuncture expectancy scale: development and preliminary validation in China. EXPLORE 2007;3:372-7.

52. Dionne CE. A standard measure of persistent bodily pain that is quick and easy to use, valid and stable over time. Pain 2012;153:1338-9.

53. Garcia MK, Driver L, Haddad R, et al. Acupuncture for treatment of uncontrolled pain in cancer patients: a pragmatic pilot study. Integr Cancer Ther 2014;13:133-40.

54. Niemtzow RC, Gambel J, Helms J, et al. Integrating ear and scalp acupuncture techniques into the care of blast-injured United States military service members with limb loss. J Altern Complement Med 2006;12:596-9.

55. Mao JJ, Bruner DW, Stricker C, et al. Feasibility trial of electroacupuncture for aromatase inhibitor--related arthralgia in breast cancer survivors. Integr Cancer Ther 2009;8:123-9.

56. Witt $\mathrm{C}$, Brinkhaus $\mathrm{B}$, Jena $\mathrm{S}$, et al. Acupuncture in patients with osteoarthritis of the knee: a randomised trial. Lancet 2005;366:136-43.

57. Huang MI, Nir Y, Chen B, et al. A randomized controlled pilot study of acupuncture for postmenopausal hot flashes: effect on nocturnal hot flashes and sleep quality. Fertil Steril 2006;86:700-10.

58. Nir Y, Huang MI, Schnyer R, et al. Acupuncture for postmenopausal hot flashes. Maturitas 2007;56:383-95.

59. Schnyer RN, Iuliano D, Kay J, et al. Development of protocols for randomized sham-controlled trials of complex treatment interventions: Japanese acupuncture for endometriosis-related pelvic pain. J Altern Complement Med 2008;14:515-22.

60. May BH, Zhang A, Lu Y, et al. The systematic assessment of traditional evidence from the premodern Chinese medical literature: a text-mining approach. J Altern Complement Med 2014;20:937-42.

61. He Y, Liu Y, May BH, et al. Effectiveness of acupuncture for cancer pain: protocol for an umbrella review and meta-analyses of controlled trials. BMJ Open 2017;7:e018494.

62. Porzsolt $F$, Eisemann $M$, Habs $M$, et al. Form follows function: pragmatic controlled trials (PCTs) have to answer different questions and require different designs than randomized controlled trials (RCTs). Z Gesundh Wiss 2013;21:307-13.

63. Mao JJ, Xie SX, Bowman MA. Uncovering the expectancy effect: the validation of the acupuncture expectancy scale. Altern Ther Health Med 2010;16:22-7.

64. Bauml J, Xie SX, Farrar JT, et al. Expectancy in real and sham electroacupuncture: does believing make it so? J Natl Cancer Inst Monogr 2014;2014:302-7. 